\title{
Correction to: The zero mass limit of Kerr and Kerr-(anti-)de-Sitter space-times: exact solutions and wormholes
}

\section{T. Birkandan ${ }^{1}$ (D) $\cdot$ M. Hortaçsu ${ }^{2}$}

Received: 25 June 2018 / Accepted: 4 August 2018 / Published online: 14 August 2018

(c) Springer Science+Business Media, LLC, part of Springer Nature 2018

\section{Correction to: Gen Relativ Gravit (2018) 50:28 https://doi.org/10.1007/s10714-018-2351-y}

Here we want to report an unfortunate misprint in the last equation, Eq. (28), in our paper The zero mass limit of Kerr and Kerr-(anti-)de-Sitter space-times: exact solutions and wormholes, which was published in General Relativity and Gravitation, [1]. Our Eq. (28) should read

$$
\begin{aligned}
& \frac{d^{2} G(v)}{d v^{2}}+\left(\frac{1}{v-\frac{3}{a^{2} \Lambda}-1}+\frac{1}{v}+\frac{1}{2\left(v-\frac{3}{a^{2} \Lambda}\right)}\right) \frac{d G(v)}{d v} \\
& +\frac{1}{v\left(v-1-\frac{3}{a^{2} \Lambda}\right)\left(v-\frac{3}{a^{2} \Lambda}\right)}\left(\frac{\left(1+\frac{3}{a^{2} \Lambda}\right) \frac{3}{4} \omega^{2}}{\Lambda v}\right. \\
& \left.+\mu^{2} a^{2}\left(v+\frac{3}{a^{2} \Lambda}\right)-\frac{m^{2}\left(1+\frac{3}{a^{2} \Lambda}\right)}{\left(v-1-\frac{3}{a^{2} \Lambda}\right)}+\lambda\right) G(v)=0 .
\end{aligned}
$$

This was the equation we got after changing our variable to $v=u+\frac{3}{a^{2} \Lambda}$ and investigated the behavior beyond the event horizon. We noted that the solution of this equation is very similar to the one given in [1], Eqs. (19-26) on page 4. We get another Heun-type solution.

The original article can be found online at https://doi.org/10.1007/s10714-018-2351-y.

$凶 \quad$ T. Birkandan

birkandant@itu.edu.tr

M. Hortaçsu

hortacsu@itu.edu.tr

1 Department of Physics, Istanbul Technical University, Istanbul, Turkey

2 Department of Physics, Mimar Sinan Fine Arts University, Istanbul, Turkey 
Here, after correcting our unfortunate misprint we take the liberty to briefly remark on the solutions of this equation. There are three regular singularities of this equation in the finite domain of the variable $v$. When $v=0$, we see that we start from this point on and go to minus infinity, for positive values of the radial variable $r$, since $v=-r^{2} / a^{2}+\frac{3}{a^{2} \Lambda}$, encountering no further singularities. When $v=\frac{3}{a^{2} \Lambda}$, we go back to $u=0$, which is before the event horizon. When $v=1+\frac{3}{a^{2} \Lambda}$, the radial coordinate will take imaginary values.

Note also that we considered only positive values of the cosmological constant in our initial paper. If we take negative values of the cosmological constant, the radial coordinate will take either values starting from zero, with no event horizon, or the radial coordinate will take imaginary values.

If we study the angular equation for negative cosmological constant, for $\frac{3}{a^{2} \Lambda}$ is greater than unity, the angular variable takes imaginary values. When $\frac{3}{a^{2} \Lambda}$ is less than unity, we get a conical singularity. When $\frac{3}{a^{2} \Lambda}=1$, we get a confluent Heun solution.

\section{Reference}

1. Birkandan, T., Hortaçsu, M., Gen. Relativ. Gravit. 50, 28 (2018) 